\title{
Primary MALT lymphoma of the colon - Pathohistological and immunohistochemical analysis, prognosis and complex treatment
}

\author{
Lena Marinova ${ }^{1 *}$; Bistra Yordanova ${ }^{2}$; Nikolay Evgeniev³ ${ }^{3}$ Ruslan Kulinski ${ }^{4}$; Albena Alkusheva ${ }^{4}$ \\ ${ }^{1}$ Complex Oncology Center, Department of Radiotherapy - Ruse, Bulgaria. \\ ${ }^{2}$ Complex Oncology Center, Department of Clinical Pathology - Ruse, Bulgaria. \\ ${ }^{3}$ Complex Oncology Center, Department of Medical Oncology - Ruse, Bulgaria. \\ ${ }^{4}$ Complex Oncology Center, Department of Surgery - Ruse, Bulgaria.
}

\section{*Corresponding Author: Lena Marinova}

Complex Oncology Center, Department of Radiotherapy - Ruse, Bulgaria.

Email: rad_marinova@abv.bg

Received: Jul 15, 2021

Accepted: Aug 26, 2021

Published: Aug 31, 2021

Archived: www.jjgastro.com

Copyright: (C) Marinova L (2021).

\section{Abstract}

Colonic MALT lymphoma is a rare disease. Due to rare diagnosis, randomized studies are missing to determine the optimal therapeutic approach. We present a 77-year-old patient with Mucosa-Associated Lymphoid Tissue (MALT) lymphoma of the colon, which is diagnosed after colonoscopy with biopsy and precise pathohistological and immunohistochemical analysis. Treatment includes surgery /hemicolonectomy, followed by 6 cycles of chemotherapy- R-COPP regimen. Two years after diagnosis, the patient is in good condition with normal quality of life without recurrence and distant spread of the disease. Through this article, our goal is to support medical literature with another clinical case that demonstrate the need for thorough and precise diagnosis by immunohistichemic analysis and an assessment of optimal complex treatment.

Keywords: MALT lymphoma; non-Hodgkin's lymphoma; pathohistological analysis; immunohistochemical analysis; chemotherapy; Complex treatment.

\section{Introduction}

In 1942, Gall and Mallory introduced a lymphoma classification based on clinicopathological criteria, the first systematic attempt to bring order into the Non-Hodgkin's Lymphoma (NHL) situation [1]. The latest WHO classification [2] recognizes 5 histological subtypes of B cell lineage: Extranodal marginal lymphoma (MALT lymphoma), follicular lymphoma, mantle cell lymphoma, diffuse large B-cell lymphoma and Burkitt's lymphoma. All histological subtypes of nodal lymphomas may arise in the gastrointestinal tract but the major two appearing in more than $90 \%$ of cases are diffuse large B-cell NHL and MucosaAssociated Lymphoid Tissue (MALT) NHL [3]. In 40\% of cases, the major site of extranodal $\mathrm{NHL}$ is the gastrointestinal tract [4]. Diffuse large B-cell lymphoma (DLBCL) is the most common $\mathrm{NHL}$ characterized by heterogeneous clinical and biological fea- tures [5]. The gross intestine is mainly involved by high grade lymphomas, in particular DLBCL (about 50\%-80\% of cases) [4]. Most involved region is sigmoid tract, followed by rectum and ascending colon [6].

\section{Clinical case}

A 77-year-old man with complaints of severe abdominal pain and obstipation. Several days after symptoms manifestation a CT of and colonoscopy with biopsy have been performed. After this assessment the patient has been diagnosed with tumor lesion in the area of the colonic splenic flexure with histological result - Mucosa-Associated Lymphoid Tissue (MALT) lymphoma of the colon with impending Immunohistochemistry (IHC). Bone marrow biopsy has been performed for further staging and has been found to be negative for any neoplastic process. 
Citation: Marinova L, Yordanova B, Evgeniev N, Kulinski R, Alkusheva A. Primary MALT Iymphoma of the colon - Pathohistological and immunohistochemical analysis, prognosis and complex treatment. Japanese J Gastroenterol Res. $2021 ; 1$ (3): 1015.

Very soon a left hemicolectomy with latero-lateral transe anastomosis has been performed. An IHC has been done to specify the histological subtype B-cell non-Hodgkin's lymphoma after that the patient is targeted for 6 cycles of chemotherapy under protocol R-COPP (rituximab with cyclophosphamide, doxorubicin, vincristine and prednisone). After two years of diagnosis, the patient is without relapse and distant spread of the disease in good quality of life.

Contrast enhancement computed tomography (CT) scan of the thorax, abdomen and pelvis

Tumor in the area of the colonic splenic flexure measuring $90 / 80 \mathrm{~mm}$. Liver, gallbladder, slender and pancreas-without pathological changes. Kidneys bilaterally-without changes. Not visualized pathologically increased paraaortal, paracaval, pelvic and inguinal lymph nodes. Lung - without pathological changes in the parenchyma of the lungs double-sided (Figure 1). There are no increased hilar, peribronchial and mediastinal lymph nodes (Figure 2).

\section{Colonoscopy with biopsy}

looked to the area of the colonic splenic flexure, where a trimming lumen tumor process is found with swelling mucosa without neoplastic changes and contact bleeding. Biopsy from different sections were taken.

\section{The result of a histological findings - Macroscopic}

Part of a thick intestine with a size of $30 \mathrm{~cm}$ with a omentum. In the middle part of the resected colon, there is a tumor formation with a diameter of $10 \mathrm{~cm}$. Eccentrically encompassing the intestine along the $10 \mathrm{~cm}$. The tumor has a whitish color and macroscopically resembles fish meat. Seven lymph nodes were removed. Microscopic -Part of a thick intestine with a tumor histologically represented by diffuse cultivated tumor cells with scarce cytoplasm, circular polymorphic nuclei with visible nucleoli and high mitotic activity / over 10 mitoses per 10 high-power fields. The wall of colon with massive, diffuse

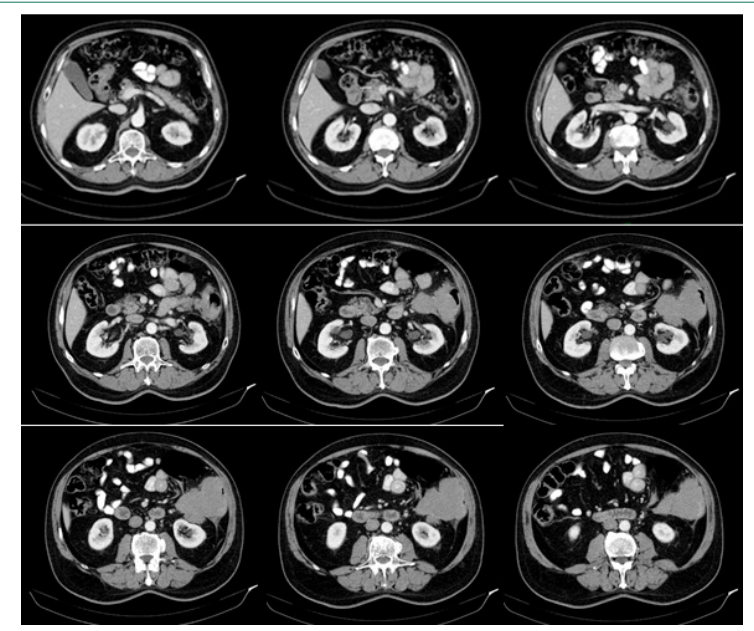

Figure 1: Contrast enhancement CT scan of the abdomen: Tumor in the area of the colonic splenic flexure measuring $90 / 80 \mathrm{~mm}$. Liver, gallbladder, slender and pancreas without pathological changes. Kidneys bilaterally-without changes. Not visualized pathologically increased paraaortal, paracaval, pelvic and inguinal lymph nodes. infiltration from large and medium-sized neoplastic cells. They have a slightly pronounced polymorphism, a dischositive way of growth, some of them with vesicular nuclei and prominent nucleoli (Figure 3A,C). Well taken out Reed-Sternberg like cells (Figure 3B). There are extensive zones with epithelium atrophy. The tumor infiltrates mucose, submucosa, smooth muscle layer, subset fat and serosis (Figure 4A). Morphology is a characteristic of MALT lymphoma. In three of the surveyed seven lymph nodes, a diffuse deletion structure was established due to tumor infiltration (Figure 4B). The resection lines are clean, without tumor infiltration. To establish the type of lymphoma, Immunohistochemical (IHC) analysis is imposed.

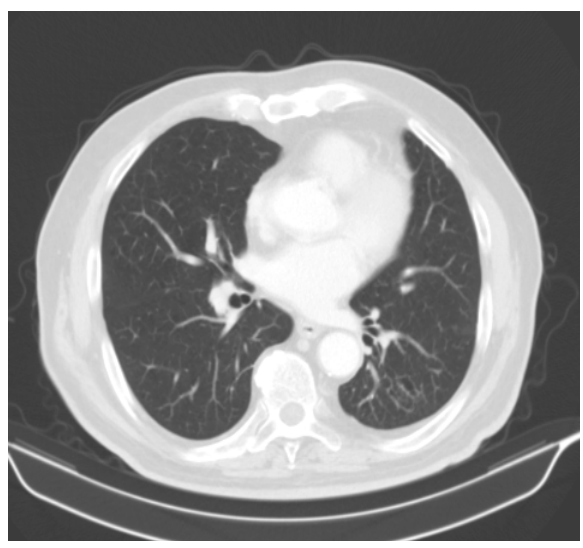

Figure 2: Contrast enhancement CT scan of the chest: Lung without pathological changes in the parenchyma of the lungs doublesided. There are no increased hilar, peribronchial and mediastinal lymph nodes.

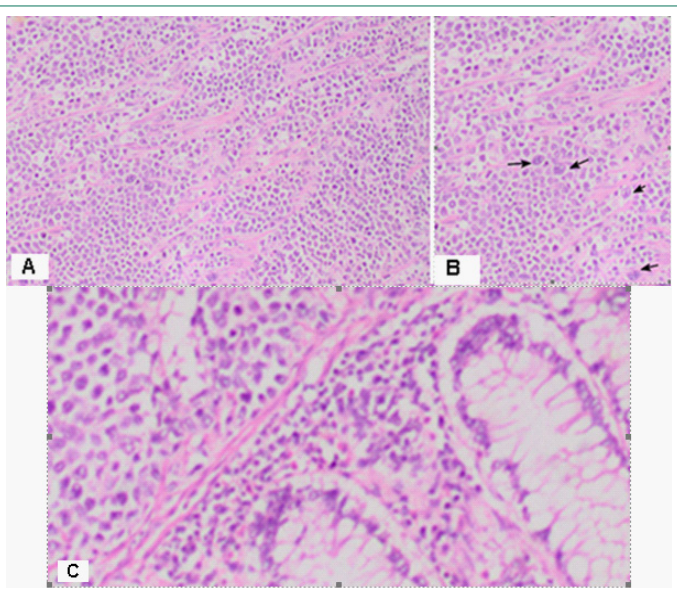

Figure 3: Microscopic histological findings: (A) The tumor histologically represented by diffuse cultivated tumor cells with scarce cytoplasm, circular polymorphic nuclei with visible nucleoli and high mitotic activity / over 10 mitoses per 10 high-power fields H\&E, x20. (B) Well taken out Reed-Sternberg like cells (mentioned with black arrow) H\&E, x20. (C) The wall of colon with massive, diffuse infiltration from large and medium-sized neoplastic cells. They have a slightly pronounced polymorphism, a dischositive way of growth, some of them with vesicular nuclei and prominent nucleoli H\&E, x200. 


\section{Immunohistochemistry (IHC)}

Positive CD20 reaction in most of the tumor population; Negative CD 3 expression in tumor cells, but positive in single stromal T lymphocytes as part of the stromal reaction; Negative Chromogranin reaction in tumor cells; Negative Vimentin expression in tumor cells, but positive in stromal lymphocytes $x 20$; Negative Synaptophysin reaction in tumor cells; Negative HMB 45 melanosome reaction in tumor cells (Figure 5A-F). Figure 6 represents the $\mathrm{IHC}$ reaction to Cytokeratins- $A$ /Negative CK 20 expression in tumor cells; B /Negative CK AE1-AE3 expression in tumor cells/positive in separate glandular structures. The metastatic three lymph nodes establish the positive CD20 and negative $\mathrm{CD} 3$ reaction (Figure 7 ).

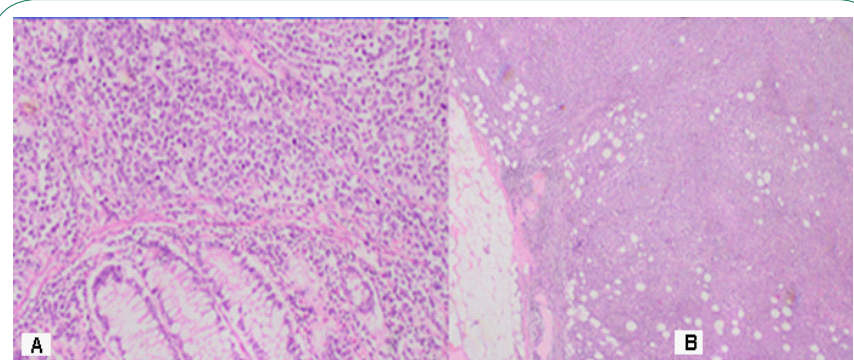

Figure 4: (A) The wall of colon with massive, diffuse infiltration from large and medium-sized neoplastic cells with slightly pronounced polymorphism, a dischositive way of growth, some of them with vesicular nuclei and prominent nucleoli H\&E, x100. (B) In three of the surveyed seven lymph nodes, a diffuse deletion structure was established due to tumor infiltration H\&E, x20.

\section{Additional IHC study}

Positive CD5 reaction in a small amount of scattered and groups of mature lymphoid cells; Positive CD10 reaction mainly in fibroblasts and fibrocytes; Positive MUM1 nuclear reaction in most of the lymphoid population; Positive $B C L 2$ reaction in most of the tumor population; Negative C-MYC reaction; Positive $\mathrm{BOB}-1$ reaction in about $70-75 \%$ of tumor cells.

Conclusion: The described immunohistochemical study corresponds to a massive transmural transmission of a large intestine wall from diffuse large $B$-cell lymphoma (DLBCL) non-GC type.

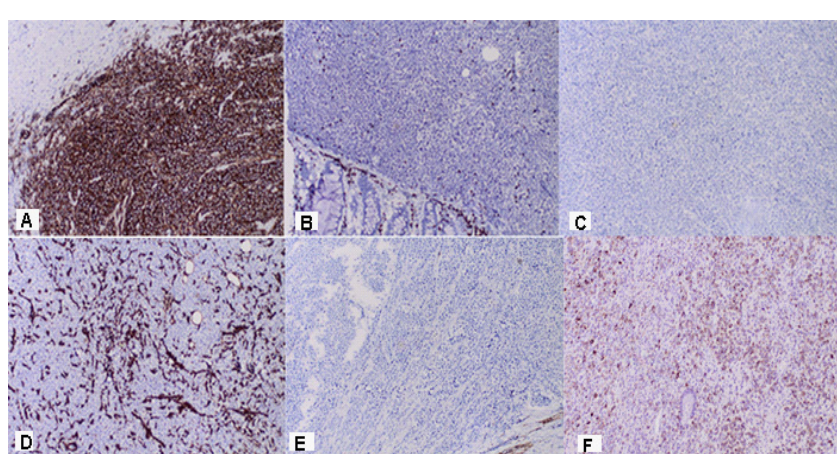

Figure 5: Immunohistochemistry - (A) Positive CD20 reaction in most of the tumor population $\times 20$; (B) Negative CD 3 expression in tumor cells, but positive in single stromal $\mathrm{T}$ lymphocytes as part of the stromal reaction $\times 20 ; C /$ Negative Chromogranin reaction in tumor cells x20; (D) Negative Vimentin expression in tumor cells, but positive in stromal lymphocytes x20; (E) Negative Synaptophysin reaction in tumor cells $\times 20$; (F) Negative HMB 45 melanosome reaction in tumor cells $\times 20$.

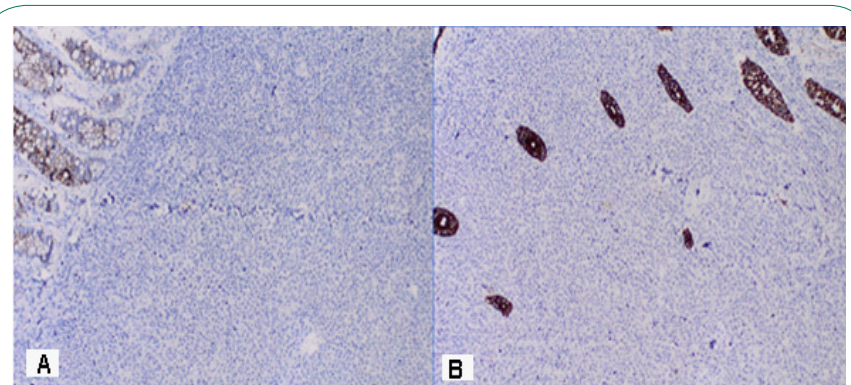

Figure 6: Immunohistochemistry: (A) Negative CK 20 expression in tumor cells $\times 20$; (B) Negative CK AE1-AE3 expression in tumor cells, but positive in the epithelium of intestinal glands $\times 20$.

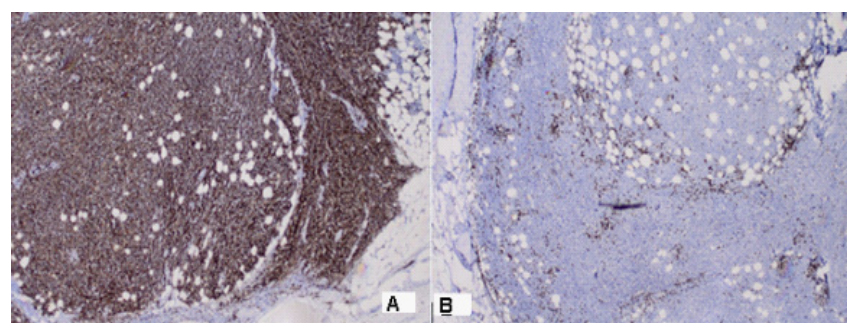

Figure 7: Immunohistochemistry: (A) Positive CD20 reaction in regional lymph nodes x20; (B) Negative CD 3 expression in metastatic tumor cells from regional lymph nodes, but positive in single T lymphocytes $\times 20$.

\section{Discussion}

MALT lymphomas are uncommon, accounting for $5 \%$ of all $\mathrm{NHL}[7,8]$ Most commonly involves gastrointestinal (GI) tract $(50 \%)$, followed in order by salivary glands, lung $(14 \%)$, head and neck (15\%), ocular adnexa (12\%), skin (11\%), thyroid (4\%) and breast (4\%) [9]. Colonic MALT Iymphoma is a rare entity, and comprises only $2.5 \%$ of MALT lymphomas [10]. Dawson et al. [11] established criteria for the diagnosis of primary colorectal lymphomas in 1961. These are: (1) no enlarged superficial lymph nodes when the patient is first seen; (2) chest radiographs without obvious enlargement of the mediastinal nodes; (3) the white blood cell counts, both total and differential, are within normal range and bone marrow biopsy is also normal; (4) at laparotomy only regional nodes are affected by disease; and (5) the liver and spleen seem free of tumor. In the modern era, these criteria have been expanded to new diagnostic tools. Krol et al. [12] in a paper from 2003 described a more liberal definition of primary extranodal NHL that includes all patients who present with NHL that apparently originated from an extranodal site, even in the presence of disseminated disease, as long as the extranodal component is clinically dominant. In $40 \%$ of cases, the major site of extranodal $\mathrm{NHL}$ is the gastrointestinal tract. Involvement of the large intestine is rare (10\%-20\% of all gastrointestinal lymphomas) in comparison to the stomach or small bowel [13]. Regarding the intestine, the ileum and the cecum are the most involved sites, probably because the abundant lymphoid tissue present [4,14]. Additionally, it represents less than $1 \%$ of malignancies affecting the gross intestine [4].

Microscopic MALT lymphoma is divided into two subtypes: (1) Low grade: Small or monocytoid lymphocytes with irregular nuclei that form lymphoepithelial lesions and (2) High grade: Resembles diffuse large B cell lymphoma but may have Reed-Sternberg like cells [15]. The term "high-grade MALT lymphoma" is confusing and should not be used. In the absence of at least a focus of unequivocal typical MALT 
Iymphoma, distinction of transformed MALT Iymphoma from DLBCL arising de novo is often impossible, particularly because the formation of lymphoepithelial lesions by DLBCL does not prove transformation from MALT Iymphoma [16]. In the publication, we present rare MALT lymphoma from colon with a immunohistochemical study corresponds to a massive transmural transmission of a large intestine wall from diffuse large B-Cell lymphoma (DLBCL).

\section{Endoscopy}

In the colon the lymphoma can arise from submucosal layers, can make the diagnosis difficult with a need to perform further endoscopic procedures [17]. Interestingly, the endoscopic pattern differ statistically between high-grade and low-grade lymphomas since high grade lymphomas have a probability to present with an exophytic mass greater than low-grade lymphomas that conversely present more frequently with petechial hemorrhages and normal/hyperemic mucosa [18]. Recently, a new endoscopic 6-items classification has been proposed [19]: (1) Exophytic: A single mass with irregular or polypoid aspect; (2) Ulcerative: Single to multiple ulcerations/erosions; (3) Hypertrophic: Enlarged folds and nodular pattern; (4) Petechial hemorrhage: Several petechiae at mucosal level; (5) Normal/ hyperemic mucosa: Normal to reddish mucosa; and (6) Mixed: more of the above-mentioned pattern present. From the molded colonoscopy, our endoscopic find is from 5 type of mucosal changes.

Computerized Tomography (CT) shows the focal form of NHL characterized by infiltrative spread rising from the submucosa, resulting in uniform thickening of the intestinal wall, usually without associated desmoplastic reaction. Infiltration of the muscularis propria and the autonomic plexus may result in atonic, aneurismal dilatation of the lumen [4]. In Figure 1, a large tumor of $90 / 80 \mathrm{~mm}$ is visualized, covering the column wall and almost clogging its lumen.

\section{Patohistological findings}

DLBCL is neoplasm of large $B$ lymphoid cells with nuclei at least $2 \times$ size of lymphocyte [20]. The pathomorphological characteristic is expressed by diffuse growth pattern with large cells (usually $5 \times$ normal lymphocytes) resembling immunoblasts (amphophilic cytoplasm, eccentric nuclei with one central nucleoli) or centroblasts (pale or basophilic cytoplasm, vesicular chromatin due to chromatin margination, 2 - 3 nucleoli, often near membrane), or rarely, anaplastic, associated with neutrophils [21]. In Figure 3 we present diffuse tumor cells with scarce cytoplasm, circular polymorphic nuclei with visible nucleoli and high mitotic activity / over 10 mitoses per 10 high-power fields. In Figure 4A is clearly seen the wall of colon with massive, diffuse infiltration from large and medium-sized neoplastic cells with slightly pronounced polymorphism, a dischositive way of growth, some of them with vesicular nuclei and prominent nucleoli. In three of the surveyed seven lymph nodes, a diffuse deletion structure was established due to tumor infiltration (Figure 4B).

Immunohistochemically, the tumor cells were positive for Bcell markers (CD20, CD10, bcl-2), which defines B cell lymphoma (Figure 5A). Cytokeratins CK20 and CK AE1-AE3 were negative in tumor cells, but positive in the epithelium of intestinal glands (Figure 6A,B). The small stromal lymphocytes were positive for Vimentin (Figure 5D). Most of the atypical lymphoid cells were negative for T-cell markers CD5 and CD3, but positive in single stromal T lymphocytes as part of the stromal reaction (Figure $5 B$ ). The positive $C D 3$ reaction in small number $T$ lymphocytes, both in the tumor and the metastatic lymph node, were part of the stromal reaction from normal lymphocytes, that are in the intestine and the lymph node (Figure 5B, 7B). The normal positive expression of $\mathrm{CD} 3$ is manifested in peripheral $T$ cells (cytoplasmic expression at early $\mathrm{T}$ cell differentiation, then membranous expression) [22]. The lymphoid tumor cells were negative for neuroendocrine marker Chromogranin and Synaptophysin (Figure 5C, E), as well as to HMB 45 melanosome (Figure $5 \mathrm{~F}$ ), which rejects the diagnosis of neuroendocrine tumor and malignant melanoma. The metastatic three lymph nodes establish the positive $\mathrm{CD} 20$ and negative CD3 reaction (Figure 7). The distinction of DLBCL according to cell of origin to germinal center (GC) and non-GC subgroups and their prognostic impact were initially investigated by gene expression profiling $[23,24]$. The subgroups can be classified immunohistochemically using a three-marker algorithm of CD10, Bcl-6 and MUM-1 has made this approach clinically more applicable. The GC and non-GC classification was determined by the Hans algorithm [25]. If CD10 was negative but Bcl-6 positive, the MUM-1 staining determined the phenotype-MUM-1 negative cases were GC- and MUM-1 positive cases were non-GC phenotype [26]. Using a three-marker algorithm of CD10, Bcl-6 and MUM-1 we have proved, that the presented diffuse large B-Cell lymphoma is non-GC phenotype.

\section{Prognosis}

Currently, the International Prognostic Index (IPI), based on five clinical variables (age, performance status, stage, extranodal involvement and lactate dehydrogenase level), is the most important tool to predict survival [27] and to classify the NHL patients into different risk groups. It was suggested that the cellular source of DLBCL relapses is of a more mature B-cell type than in low-grade B-cell malignancies [28]. These observations are concordant with the fact that, compared with other types of non-Hodgkin lymphoma, de novo DLBCL rarely shows chronicity and late relapses. Indeed, 2 years after successful first-line chemotherapy for DLBCL, the relapse rate is low and overall survival is similar to the general population [29]. Several prospective trials have shown that adding rituximab to standard $\mathrm{CHOP}$ regiment ( $\mathrm{R}-\mathrm{CHOP}$ ) resulted in higher response rates and better progression-free, event-free, disease-free and overall survival [30].

\section{Complex treatment}

The optimal management of primary lymphoma of the colon and rectum has never been determined by randomized trials [4], remains uncertain and limited largely to small retrospective series or case reports [31]. Due to a delay in diagnosis in $33 \%-65 \%$ of patients, operative procedure is either urgent or emergent [32-34]. In more than half of patients, lymphoma is a bulky disease reaching over $5 \mathrm{~cm}$ in diameter [34-36]. In the published studies, treatment varies from chemotherapy alone to multimodal therapies combining surgery, chemotherapy and radiotherapy [4]. It is believed that surgery may provide important prognostic information, including history, tumor extent and stage, could prevent complications and may offer a chance for cure with or without chemotherapy [36]. ]. The chemotherapy regimen comprised of mitoxantrone, chlorambucil, and prednisone has been used to successfully treat colonic MALT lymphoma $[37,38]$. Some authors proposed the so called preventive chemotherapy in patients with stage IE, reaching five year survival of $80 \%$ [39]. Chemotherapy, as well as surgical resec- 
tion, has been used to successfully achieve cure [40]. The CHOP chemotherapeutic regimen (cyclophosphamide, doxorubicin, vincristine and prednisone) remains the first line therapy for all moderate and high-grade B-cell lymphomas [4]. Interestingly, addition of rituximab to first line chemotherapy also eliminates the adverse impact of non-GC phenotype for survival [41]. In our clinical case after a radical operation and 6 cycles of chemotherapy under R-COPP regimen, we achieved two years diseasefree survival with good quality of life.

\section{Conclusion}

The colonic MALT lymphoma is a rare disease. To resolve this case, a colonoscopy with biopsy, CT-image with intravenous contrast, bone marrow biopsy and precisely pathohistological and immunohistochemical study have been required. Due to rare diagnosis, randomized studies are missing to determine the optimal therapeutic approach. In our clinical case after a radical operation and 6 cycles of chemotherapy under R-COPP regimen, we achieved two years progression free survival with good quality of life. Through this article, our goal is to support medical literature with another clinical case that demonstrate the need for thorough and precise diagnosis by immunohistichemic analysis and an assessment of optimal complex treatment.

\section{References}

1. Aisenberg AC. Historical review of lymphomas. $\mathrm{Br} \mathrm{J}$ Haematol. 2000; 109:466-476.

2. Harris NL, Jaffe ES, Diebold J, et al. Lymphoma classificationfrom controversy to consensus: the R.E.A.L. and WHO Classification of lymphoid neoplasms. Ann Oncol. 2000; 11: 3-10.

3. Liang R, Todd D, Ho FC. Aggressive non-Hodgkin's lymphoma: T-cell versus B-cell. Hematol Oncol. 1996; 14: 1-6.

4. Goran Z Stanojevic, Milica D Nestorovic, Branko R Brankovic, et al. Primary colorectal lymphoma: An overview. World J Gastrointest Oncol. 2011; 3: 14-18.

5. Lossos IS. Molecular pathogenesis of diffuse large B-cell lymphoma. J Clin Oncol. 2005; 23: 6351-6357.

6. Terada T. Gastrointestinal malignant lymphoma: A pathologic study of 37 cases in a single Japanese institution. Am J Blood Res. 2012; 2: 194-200.

7. Romaguera J, Hagemeister FB. Lymphoma of the colon. Curr Opin Gastroenterol. 2005; 21: 80-84.

8. Hasegawa N, Kato K, Yamada K, et al. Extranodal marginal zone $B$-cell lymphoma of mucosa-associated lymphoid tissue (MALT) of the sigmoid colon. Gastrointest Endosc. 2000; 52: 802-4.

9. Tadashi Terada. Extranodal marginal zone lymphoma of mucosaassociated lymphoid tissue (MALT Iymphoma) of the ileum in a 35-year-old Japanese woman. Int J Clin Exp Pathol. 2013; 6: 951-956.

10. Chen PH, Lin YM, Yen HH. Primary mucosa-associated lymphoid tissue lymphoma of the colon. Clin Gastroenterol Hepatol. 2011; 9: e74-75.

11. Dawson IM, Cornes JS, Morson BC. Primary malignant lymphoid tumours of the intestinal tract. Report of 37 cases with a study of factors influencing prognosis. Br J Surg. 1961; 49: 80-89.

12. Krol AD, le Cessie $S$, Snijder $S$, et al. Primary extranodal nonHodgkin's lymphoma (NHL): The impact of alternative definitions tested in the Comprehensive Cancer Centre West population-based NHL registry. Ann Oncol. 2003; 14: 131-139.
13. Crump M, Gospodarowicz M, Shepherd FA. Lymphoma of the gastrointestinal tract. Semin Oncol. 1999; 26: 324-337.

14. Ghimire P, Wu GY, Zhu L. Primary gastrointestinal lymphoma. World J Gastroenterol. 2011; 17: 697-707.

15. Gulwani H. MALT lymphoma. PathologyOutlines.com. 2021.

16. Chris M Bacon, Ming-Qing Du, and Ahmet Dogan. Mucosa-associated lymphoid tissue (MALT) lymphoma: a practical guide for pathologists. J Clin Pathol. 2007; 60: 361-372.

17. Ikuta K, Fujiya M, Ueno N, et al. Atypical mucosa-associated lymphoid tissue lymphoma in the transverse colon associated with macroglobulinemia. Intern Med. 2010; 49: 677-682.

18. Zullo A, Hassan C, Andriani A, et al. Primary low-grade and highgrade gastric MALT-lymphoma presentation. J Clin Gastroenterol. 2010; 44: 340-344.

19. Zullo A, Hassan C, Cristofari F, et al. Gastric low-grade mucosalassociated lymphoid tissue-lymphoma: Helicobacter pylori and beyond. World J Gastrointest Oncol. 2010; 2: 181-186.

20. Sangle N. DLBCL-NOS. PathologyOutlines.com. 2021.

21. S Badve, A Blumstein, P Wiernik, H Ratech. Non-hodgkin malignant lymphoma with tissue neutrophilia: A report of 3 cases. 2000; 124: 735-8.

22. Pernick N. CD3. PathologyOutlines.com website. https://www. pathologyoutlines.com/topic/cdmarkerscd3.html.

23. Alizadeh AA, Eisen MB, Davis RE, et al. Distinct types of diffuse large B-cell lymphoma identified by gene expression profiling. Nature. 2000; 403: 503-511.

24. Rosenwald A, Wright G, Chan WC, et al. The use of molecular profiling to predict survival after chemotherapy for diffuse largeB-cell lymphoma. N Engl J Med. 2002; 346: 1937-1947.

25. Hans CP, Weisenburger DD, Greiner TC, et al. Confirmation of the molecular classification of diffuse large B-cell lymphoma by immunohistochemistry using a tissue microarray. Blood. 2004; 103: 275-282.

26. H Nyman, E Jantunen, E Juvonen, et al. Impact of germinal center and non-germinal center phenotypes on overall and failure-free survival after high-dose chemotherapy and auto-SCT in primary diffuse large B-cell lymphoma. Bone Marrow Transplantation. 2008; 42: 93-98.

27. IPI-Project. A predictive model for aggressive non-Hodgkin's lymphoma. The International Non-Hodgkin's Lymphoma Prognostic Factors Project. N Engl J Med. 1993; 329: 987-994.

28. Simon Husby and Kirsten Gronbæk. Mature lymphoid malignancies: origin, stem cells, and chronicity. Blood Adv. 2017 ; 1: 2444-2455.

29. Maurer MJ, Ghesquières $H$, Jais J-P, et al. Event-free survival at 24 months is a robust end point for disease-related outcome in diffuse large B-cell lymphoma treated with immunochemotherapy. J Clin Oncol. 2014; 32: 1066-1073.

30. Morrison VA. Evolution of R-CHOP therapy for older patients with diffuse large B-cell lymphoma. Expert Rev Anticancer Ther. 2008; 8: 1651-1658.

31. Schwartz Brian L, Lowe Robert C. Successful Endoscopic Resection of Mucosa-Associated Lymphoid Tissue Lymphoma of the Colon. ACG Case Reports Journal. 2019; 6: 00228.

32. Stanojević $\mathrm{G}$, Stojanović $\mathrm{M}$, Jovanović $\mathrm{M}$, et al. [Primary colorectal lymphomas] Vojnosanit Pregl. 2009;66:295-301. 
33. Cai S, Cannizzo F Jr, Bullard Dunn KM, et al. The role of surgical intervention in non-Hodgkin's lymphoma of the colon and rectum. Am J Surg. 2007; 193: 409-412.

34. Bairey O, Ruchlemer R, Shpilberg O. Non-Hodgkin's lymphomas of the colon. Isr Med Assoc J. 2006; 8: 832-835.

35. Stanojević GZ, Stojanović MP, Stojanović MM, et al. Non-Hodgkin's lymphomas of the large bowel-clinical characteristics, prognostic factors and survival. Acta Chir lugosl. 2008; 55: 109-114.

36. Fan CW, Changchien CR, Wang JY, et al. Primary colorectal lymphoma. Dis Colon Rectum. 2000; 43: 1277-1282.

37. Gezen C, Kement M, Oncel M, et al. Mucosa associated lymphoid tissue lymphoma of the colon: A case report. Cases J. 2009; 2: 9316.
38. Sallach S, Schmidt T, Pehl C, et al. Primary low-grade B cell nonHodgkin's lymphoma of MALT type simultaneously arising in the colon and in the lung: Report of a case. Dis Colon Rectum. 2001; 44: 448-52.

39. Devine R, Brand M. Miscellaneous Neoplasms. In: Wolff B, Fleshman J, Beck D, Pemberton J, Wexner S, editors. The ASCRS textbook of colon and rectal surgery. Springer; 2007; 515-524.

40. Hafsa Abbas, Masooma Niazi, Jasbir Makker. Mucosa-Associated Lymphoid Tissue (MALT) Lymphoma of the Colon: A Case Report and a Literature Review. Am J Case Rep. 2017; 18: 491-497.

41. Nyman $\mathrm{H}$, Adde M, Karjalainen-Lindsberg ML, et al. Prognostic impact of immunohistochemically defined germinal center phenotype in diffuse large B-cell lymphoma patients treated with immunochemotherapy. Blood. 2007; 109: 4930-4935. 